\title{
Prognostic implications of serum lipid metabolism over time during sepsis
}

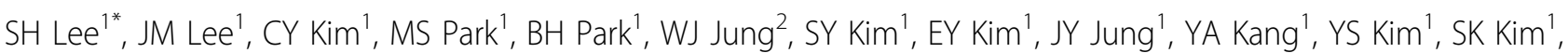 \\ J Chang ${ }^{1}$, KS Chung ${ }^{1}$
}

From ESICM LIVES 2015

Berlin, Germany. 3-7 October 2015

\section{Intr}

Despite extensive research and an improved standard of care, sepsis remains a disorder with a high mortality rate. Sepsis is accompanied by severe metabolic alterations. However, there are controversies about changes in lipid profiles in sepsis patients.

\section{Objectives}

To examine whether disorders of lipid metabolism are associated with worse disease activity and mortality.

\section{Methods}

We evaluated 117 sepsis and septic shock patients (65 survivors and 52 non-survivors) who were admitted to the intensive care unit of a university-affiliated hospital in Korea. Serum levels of cholesterol, triglyceride (TG), high-density lipoprotein (HDL), low-density lipoprotein (LDL), free fatty acid (FFA), and apolipoprotein (apo) A-I were measured on days 0, 1, 3, and 7. Patients who had previously used statins or steroids, for treating lipid diseases or liver diseases were excluded.

\section{Results}

Non-survivors showed significantly higher SOFA scores than survivors $(p=0.008)$. Non-survivors had low levels of cholesterol, TG, HDL, LDL, and apo A-I levels on days 0 , 1,3 , and 7. In linear mixed model analysis, over time the variation in TG, LDL, FFA and apo A-I between groups differed significantly $(\mathrm{p}=0.043, \mathrm{p}=0.020, \mathrm{p}=0.005$, and $\mathrm{p}=0.015$, respectively). However, there were no significant differences in cholesterol or HDL between groups. Through multivariate analysis, TG level and SOFA scores were associated with mortality on day 0 and day $1(\mathrm{p}=0.018$ and

'Severance Hospital, Yonsei University College of Medicine, Seoul, Korea, Republic of Korea

Full list of author information is available at the end of the article $p=0.008$, respectively). In survival analysis, the high TG level group showed better prognosis than the other groups $(\mathrm{p}=0.022)$.

\section{Conclusions}

Recently, in two large, multicenter, double-blind, randomized, placebo-controlled studies, simvastatin and rosuvastatin did not improve clinical outcomes in acute respiratory distress syndrome (ARDS) or sepsis-associated ARDS patients. Similary, in contrast to previous studies, our study showed that low TG level is associated with mortality in ICU patients with sepsis. This may be due to alterations in serum lipid metabolism during sepsis, modulating the host response to inflammation in critically ill patients.

\section{Authors' details}

'Severance Hospital, Yonsei University College of Medicine, Seoul, Korea, Republic of Korea. ${ }^{2}$ Korea University Anam Hospital, Korea University College of Medicine, Seoul, Korea, Republic of Korea.

Published: 1 October 2015

\section{References}

1. Mortality related to severe sepsis and septic shock among critically ill patients in Australia and New Zealand, 2000-2012. JAMA 2014, 311:1308-1316.

2. Lipoproteins in inflammation and sepsis. I. Basic science. Intensive Care Medicine 2007, 33:13-24.

3. Lipoproteins in inflammation and sepsis. II. Clinical aspects. Intensive Care Med 2007, 33:25-35.

4. Lipoprotein metabolism in patients with severe sepsis. Critical Care Medicine 2003, 31:1359-1366.

5. Rosuvastatin for Sepsis-Associated Acute Respiratory Distress Syndrome. New England Journal of Medicine 2014, 370:2191-2200.

6. Simvastatin in the Acute Respiratory Distress Syndrome. New England Journal of Medicine 2014, 371:1695-1703.

doi:10.1186/2197-425X-3-S1-A226

Cite this article as: Lee et al:: Prognostic implications of serum lipid metabolism over time during sepsis. Intensive Care Medicine Experimental 2015 3(Suppl 1):A226.

\section{SpringerOpen $^{\odot}$}

(c) 2015 Lee et al.; This is an Open Access article distributed under the terms of the Creative Commons Attribution License (http:// creativecommons.org/licenses/by/4.0), which permits unrestricted use, distribution, and reproduction in any medium, provided the original work is properly cited. 